\title{
On the Existence of Berge Equilibrium: An Order Theoretic Approach
}

\author{
Kerim Keskin* and H. Çağrı Sağlam \\ Department of Economics, Bilkent University \\ 06800 Ankara, Turkey \\ *kerim@bilkent.edu.tr. \\ Received 21 August 2013 \\ Revised 6 June 2014 \\ Accepted 9 June 2014 \\ Published 14 July 2015
}

\begin{abstract}
We propose lattice-theoretical methods to analyze the existence and the order structure of Berge equilibria (in the sense of Zhukovskii) in noncooperative games. We introduce Berge-modular games, and prove that the set of Berge equilibrium turns out to be a complete lattice.
\end{abstract}

Keywords: Berge equilibrium; Berge equilibrium in the sense of Zhukovskii; supermodularity; games with strategic complementarities; Berge-modular games; fixed point theory.

JEL Classification: C62, C72

\section{Introduction}

In this paper, we propose lattice-theoretical methods to analyze the existence and the order structure of Berge equilibria (in the sense of Zhukovskii) in noncooperative games [Berge, 1957; Zhukovskii, 1994]. At a Berge equilibrium, each agent's payoff is maximized by the complementary coalition formed by all the other players. Given that a player chooses her strategy from a Berge equilibrium, among the strategy profiles of all the remaining players, the strategy profile from this particular equilibrium will yield her the highest payoff. Indeed, if an agent deviates from her Berge equilibrium strategy, the remaining agents become worse off, whereas the payoff for the deviating agent may increase or decrease. This reflects some of the common motivations in human behavior that influence strategy choices such as altruism, reciprocation, cooperation, and coordination. ${ }^{\text {a }}$

a See Güth et al. [1982], Forsythe et al. [1994], Fehr and Schmidt [1999], Bolton and Ockenfels [2000], Charness and Rabin [2002], Arnsperger and Varoufakis [2003], for the emerging recognition of such motivations in game theory. 
Berge equilibrium provides an alternative solution for games that do not have Nash equilibrium or that have multiple Nash equilibria. In contrast to Nash equilibrium where players take into account only own interests, Berge equilibrium models a type of altruism under which it is possible to reach cooperative features in a noncooperative framework. In particular, Berge equilibrium advocates reasonable solutions to the social dilemmas generated by Nash equilibrium (see Musy et al. [2012]) and to the coordination failures in the common interest games (see Colman et al. [2011]).

The set of Berge equilibrium can be defined as the set of fixed points of the joint best response correspondence, ${ }^{\mathrm{b}}$ which is defined in a different manner than the standard Nash equilibrium arguments. Although one can always obtain a nonempty-valued joint best response correspondence by taking the direct product of nonempty-valued individual best response correspondences according to Nash equilibrium, this is not the case for Berge equilibrium. According to Berge equilibrium, the intersection of coalitional best response correspondences defines the joint best response correspondence which could be in general empty. Therefore, to ensure the existence of Berge equilibrium, there exist two main challenges: (i) the nonemptyvaluedness of the joint best response correspondence; and (ii) the existence of a fixed point of the joint best response correspondence.

In this paper, we first provide a sufficient condition under which the joint best response correspondence is nonempty-valued. Once the nonempty-valuedness is guaranteed, then the problem actually reduces to a fixed point argument.

Since the notion of Berge equilibrium emphasizes the coalitions of players and induces more coordination than competition with altruistic orientation, it has much to offer under strategic complementarities. Because of this, in contrast to the earlier contributions on the existence of Berge equilibrium that are based on topologically oriented approaches, ${ }^{\mathrm{c}}$ we resort to lattice-theoretical methods that exploit the order and monotonicity properties of games. In particular, we introduce Berge-modular games in which the strategy space is a complete lattice, and the joint best response correspondence is nonempty-valued and Veinott-increasing. By means of Zhou's [1994] extension of Tarski [1955] fixed point theorem to set-valued maps, we prove that the set of Berge equilibrium turns out to be a complete lattice. Moreover, we refer to Echenique [2005] for a construction toward the extremal equilibria, and to Topkis [1998] for a monotone comparative statics result on the equilibrium set.

The paper is structured as follows: In Sec. 2, we give the definitions and theorems that are used throughout this study. Section 3 includes the results on the set of Berge equilibrium. Section 4 concludes.

\footnotetext{
${ }^{\mathrm{b}}$ In this paper, best response correspondences are according to Berge equilibrium unless otherwise stated.

${ }^{\mathrm{c}}$ Abalo and Kostreva [2004, 2005] study the existence of a more general version of Berge equilibrium for S-equi-well-posed games. Thereafter, Nessah et al. [2007] and Larbani and Nessah [2008] improve the existence results.
} 


\section{Preliminaries}

A partially ordered set is a lattice if it contains the supremum and the infimum of each pair of its elements. A lattice is complete if each nonempty subset has a supremum and an infimum. A subset $Y$ of a lattice $X$ is a subcomplete sublattice of $X$ if for each nonempty subset $Y^{\prime}$ of $Y$, the supremum $\bigvee_{X} Y^{\prime}$ and the infimum $\bigwedge_{X} Y^{\prime}$ exist, and are contained in $Y$.

Let $X$ be a lattice. A function $f: X \rightarrow \mathbb{R}$ is upper semi-continuous if for every $x \in X$ and every sequence $\left(x_{t}\right) \rightarrow x$, we have $\lim \sup f\left(x_{t}\right) \leq f(x)$. Letting $x \vee y$ $(x \wedge y)$ denote the least (greatest) element that is greater (less) than or equal to $x$ and $y$, we say that $f$ is supermodular if $\forall x, y \in X$ :

$$
f(x)+f(y) \leq f(x \wedge y)+f(x \vee y) .
$$

Let $T$ be a partial order, and define a function $f$ on $X \times T$. The function $f$ has increasing differences in $(x, t)$ if $f\left(x, t^{\prime}\right)-f(x, t)$ is increasing in $x$ for every $t<t^{\prime}$. A multi-valued map $F: X \rightarrow X$ is Veinott-increasing if for each $x, y \in X$ with $x<y, a \in F(x)$ and $b \in F(y)$ imply $a \wedge b \in F(x)$ and $a \vee b \in F(y)$. Finally, the set of fixed points of a multi-valued map $F: X \rightarrow X$ is defined as $\{x \in X \mid x \in F(x)\}$.

In addition, below are the theorems we refer to throughout this paper:

Theorem 1 ([Zhou, 1994]). Let $X$ be a nonempty complete lattice, and $F: X \rightarrow X$ be a nonempty-valued multi-valued map. If $F$ is Veinott-increasing, and $F(x)$ is a subcomplete sublattice of $X$ for every $x \in X$, then the fixed point set of $F$ is a nonempty complete lattice.

Theorem 2 ([Topkis, 1998]). Let $X$ be a nonempty complete lattice, $T$ be a partially ordered set, and $Y: X \times T \rightarrow X \times T$ be a multi-valued map. If $Y$ is Veinott-increasing, and $Y(x, t)$ is a nonempty subcomplete sublattice of $X \times T$ for every $(x, t)$, then

(i) $\forall t \in T$, there exists a greatest (least) fixed points of $Y(x, t)$;

(ii) the greatest (least) fixed point of $Y(x, t)$ is increasing in $t$ on $T$.

\section{Main Results}

In this section, to show the existence of Berge equilibrium, we use an order theoretic approach from the literature on games with strategic complementarities (GSC) in which the joint best response correspondence is Veinott-increasing. We further propose sufficient conditions on utility functions for a game to have Berge equilibrium, in a similar way supermodular games are suggested for the existence of Nash equilibrium.

Let $G=\left(N,\left(X_{i}\right)_{i \in N},\left(u_{i}\right)_{i \in N}\right)$ be a normal form game such that $N$ is the set of agents, $X_{i}$ is the strategy set of agent $i$, and $u_{i}$ is the utility function for agent $i$. The set of strategy profiles $\prod_{i \in N} X_{i}$ is denoted by $X$ with a generic element $x=\left(x_{i}, x_{-i}\right)$ where $x_{i} \in X_{i}$ and $x_{-i} \in X_{-i} \equiv \prod_{j \in N \backslash\{i\}} X_{j}$. 
The following definition of Berge equilibrium in the sense of Zhukovskii is due to Larbani and Nessah [2008].

Definition 1. In a game $G$, a strategy profile $x \in X$ is a Berge equilibrium if $\forall i \in N: u_{i}(x) \geq u_{i}\left(x_{i}, y_{-i}\right)$ for every $y_{-i} \in X_{-i}$.

For every agent $i \in N$, the complementary coalition, $-i$, has the coalitional best response correspondence $B_{-i}: X \rightarrow X$ which is defined as:

$$
B_{-i}(x)=\left\{y \in X \mid \forall y^{\prime} \in X: u_{i}\left(x_{i}, y_{-i}\right) \geq u_{i}\left(x_{i}, y_{-i}^{\prime}\right)\right\} .
$$

We define the joint best response correspondence $B: X \rightarrow X$ as follows:

$$
B(x)=\bigcap_{i \in N} B_{-i}(x)
$$

Note that the set of fixed points of $B$ turns out to be the set of Berge equilibrium. ${ }^{\mathrm{d}}$ Before proceeding to the existence result, we provide two examples in which the importance of Berge equilibrium is highlighted. In the first example, all of the strategy profiles are Nash equilibria. However, there is a unique Berge equilibrium which yields the Pareto optimal outcome: $\left(y_{1}, y_{2}\right)$.

\begin{tabular}{l|c|c|}
$y_{1}$ & 0,2 & 2,2 \\
\cline { 2 - 3 }$x_{1}$ & 0,0 & 2,0 \\
\cline { 2 - 3 } & \multicolumn{1}{c|}{$x_{2}$} & $y_{2}$
\end{tabular}

In the second example, there exists no pure strategy Nash equilibrium, but there is a unique Berge equilibrium: $\left(z_{1}, z_{2}\right)$.

\begin{tabular}{l|c|c|c|}
\cline { 2 - 4 }$z_{1}$ & 0,0 & 0,1 & 1,2 \\
\cline { 2 - 4 }$y_{1}$ & 1,1 & 1,0 & 2,0 \\
\cline { 2 - 4 }$x_{1}$ & 2,0 & 2,0 & 1,1 \\
\cline { 2 - 4 } & \multicolumn{1}{c}{$x_{2}$} & $y_{2}$ & $z_{2}$
\end{tabular}

As for the existence result, we first modify the definition of GSC by using the definition of coalitional best response correspondences. ${ }^{\mathrm{e}}$

Definition 2. A game $G$ has strategic complementarities à la Berge (or, is a GSC à la Berge) if (i) every $X_{i}$ is a nonempty, complete lattice; (ii) $B$ is nonemptyvalued and Veinott-increasing in $x$ on $X$; and (iii) $\forall x \in X, B(x)$ is a subcomplete sublattice of $X$.

Sufficient conditions for a normal form game to be a GSC are given as follows: (i) every $X_{i}$ is a nonempty, compact, and complete lattice; ${ }^{\mathrm{f}}$ (ii) each $u_{i}$ is supermodular

\footnotetext{
d To prove this, take a fixed point of the correspondence; say $x \in X$. Then $\forall i \in N: x \in B_{-i}(x)$. That is to say $\forall i \in N, \forall y_{-i} \in X_{-i}: u_{i}(x) \geq u_{i}\left(x_{i}, y_{-i}\right)$; i.e., $x$ is a Berge equilibrium. And, the converse similarly follows.

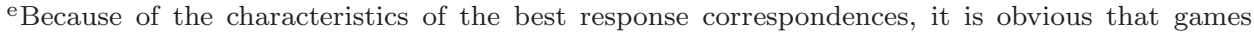
defined below differ from GSC (see Vives [2005] for a definition).

${ }^{\mathrm{f}}$ Note that a complete lattice is already compact in its interval topology which is the topology generated by taking the closed intervals as a subbasis of closed sets.
} 
in $x_{i}$, and has increasing differences in $\left(x_{i}, x_{-i}\right)$; and (iii) each $u_{i}$ is upper semicontinuous in $x_{i}$. A normal form game that satisfies all of these conditions is said to be a supermodular game. Noting that a supermodular game is not necessarily a GSC à la Berge, we introduce new classes of games. Our analysis starts with two-player games.

Definition 3. A game $G$ is opponent-wise supermodular if (i) each $X_{i}$ is a nonempty, compact, and complete lattice; (ii) each $u_{i}$ is supermodular in $x_{-i}$, and has increasing differences in $\left(x_{i}, x_{-i}\right)$; and (iii) each $u_{i}$ is upper semi-continuous in $x_{-i}$.

Theorem 3. A two-player opponent-wise supermodular game is a GSC à la Berge. A GSC à la Berge has a Berge equilibrium. Moreover, the equilibrium set is a complete lattice.

Proof. First, every coalitional best response correspondence $B_{-i}$ is nonemptyvalued since each $X_{i}$ is compact, and each $u_{i}$ is upper semi-continuous in $x_{-i}$. As we consider two-player games, the joint best response correspondence $B$ is nonemptyvalued as well.

For Veinott-increasingness, we need to show: $\forall x, y \in X$ with $x<y$, if $a \in B(x)$ and $b \in B(y)$ then $a \wedge b \in B(x)$ and $a \vee b \in B(y)$. Take any $x, y \in X$ such that $x<y$. Then take any $a \in B(x)$ and $b \in B(y)$. If $a \leq b$, we are trivially done. Consider any of the other cases: We either have $a>b$, or we cannot compare $a$ and $b$. Since $\forall i \in N: a \in B_{-i}(x)$ and $b \in B_{-i}(y)$, we have

$$
\begin{aligned}
0 \leq u_{i}\left(x_{i}, a_{-i}\right)-u_{i}\left(x_{i}, a_{-i} \wedge b_{-i}\right) & \leq u_{i}\left(x_{i}, a_{-i} \vee b_{-i}\right)-u_{i}\left(x_{i}, b_{-i}\right) \\
& \leq u_{i}\left(y_{i}, a_{-i} \vee b_{-i}\right)-u_{i}\left(y_{i}, b_{-i}\right) \leq 0 .
\end{aligned}
$$

Here, the first and the last inequalities follow from optimality. Supermodularity implies the second inequality, and increasing differences implies the third inequality. It is obvious that each term is 0 which leads to $a \wedge b \in B_{-i}(x)$ and $a \vee b \in B_{-i}(y)$. Since $i$ is arbitrary, $a \wedge b \in B(x)$ and $a \vee b \in B(y)$. Thus, $B$ is Veinott-increasing.

For increasing differences property, take any $x \in X$. Also, pick any $a, b \in B(x)$. By definition, $\forall i \in N: a, b \in B_{-i}(x)$, that is

$$
u_{i}\left(x_{i}, a_{-i}\right)=u_{i}\left(x_{i}, b_{-i}\right) \geq u_{i}\left(x_{i}, x_{-i}^{\prime}\right), \quad \forall x_{-i}^{\prime} \in X_{-i} .
$$

Moreover,

$$
u_{i}\left(x_{i}, a_{-i}\right)+u_{i}\left(x_{i}, b_{-i}\right) \leq u_{i}\left(x_{i},(a \wedge b)_{-i}\right)+u_{i}\left(x_{i},(a \vee b)_{-i}\right)
$$

by supermodularity. Now, using the optimality of $a$ and $b$, we conclude that $a \wedge b$ and $a \vee b$ are also in $B_{-i}(x)$. Trivially, they are also elements of $B(x)$. Hence for every $x \in X, B(x)$ is a subcomplete sublattice of $X$. This completes the proof that the game is a GSC à la Berge. Then $B$ satisfies the assumptions of Theorem 1 . That is to say, the set of Berge equilibrium is a nonempty complete lattice. 
On the contrary, opponent-wise supermodularity does not suffice for the existence of Berge equilibrium for games with more than two players, because the nonempty-valuedness of the joint best response correspondence may not be satisfied. ${ }^{g}$ This obviously prevents the application of Zhou's [1994] fixed point theorem. However, any additional assumption that states or implies the nonempty-valuedness of the joint best response correspondence would complete the sufficient conditions for the existence of Berge equilibrium. Among several alternatives, we prefer to utilize symmetry in preference orders. First reason is that there are many examples of environments in the literature on GSC satisfying symmetry in preference orders; such as aggregative games and coordination games. As a matter of fact, the set of games is even wider if one steps out of the boundaries of GSC. Second, symmetry in preference orders indicates that agents are identical in a sense, and considering that individuals tend to care for others who are similar to them, it is perfectly convenient for studying an equilibrium notion which incorporates altruism.

For the definition below, it is assumed for every $i \in N$ that $X_{i}=Y$. Then for every $i, j \in N$, we define a transformation $T_{i, j}: Y^{|N|} \rightarrow Y^{|N|}$ such that

$$
\begin{aligned}
& T_{i, j}\left(x_{1}, \ldots, x_{i-1}, a, x_{i+1}, \ldots, x_{j-1}, b, x_{j+1}, \ldots, x_{|N|}\right) \\
& \quad=\left(x_{1}, \ldots, x_{i-1}, b, x_{i+1}, \ldots, x_{j-1}, a, x_{j+1}, \ldots, x_{|N|}\right) .
\end{aligned}
$$

Moreover, let $L(x, \cdot)$ denote the lower contour set of $x \in X$.

Definition 4. The agents $i, j \in N$ are symmetric in preference orders if for every $x \in X$ :

$$
T_{i, j}(y) \in L\left(x, u_{i}\right) \Rightarrow y \in L\left(T_{i, j}(x), u_{j}\right)
$$

Definition 5. A game $G$ is Berge-modular if (i) each $X_{i}$ is a nonempty, compact, and complete lattice; (ii) each $u_{i}$ is supermodular in $x_{-i}$, and has increasing differences in $\left(x_{i}, x_{-i}\right)$; (iii) each $u_{i}$ is upper semi-continuous in $x_{-i}$; and (iv) all agents are pairwise symmetric in preference orders.

Theorem 4. A Berge-modular game is a GSC à la Berge; thus, has a Berge equilibrium. Moreover, the equilibrium set is a complete lattice.

gWhen there are more than two players, the nonempty-valuedness of $B_{-i}$ for every $i \in N$ does not suffice for the nonempty-valuedness of $B$ as it would have been the case for individual and joint best response correspondences according to Nash equilibrium. Here is an example in which the utility function $u_{i}$ is supermodular in $x_{-i}$, and has increasing differences in $\left(x_{i}, x_{-i}\right)$ for every agent $i$.

Let $N=\{1,2,3\}$, and $X_{i}=\left\{x_{i}, y_{i}\right\}$ with $x_{i} \leq y_{i}$ for every $i \in N$. Define $u_{1}: X \rightarrow \mathbb{R}$ such that $u_{1}\left(x_{1}, x_{2}, x_{3}\right)=11, u_{1}\left(y_{1}, x_{2}, x_{3}\right)=u_{1}\left(x_{1}, y_{2}, x_{3}\right)=u_{1}\left(x_{1}, x_{2}, y_{3}\right)=7, u_{1}\left(x_{1}, y_{2}, y_{3}\right)=$ $u_{1}\left(y_{1}, y_{2}, x_{3}\right)=u_{1}\left(y_{1}, x_{2}, y_{3}\right)=3$, and $u_{1}\left(y_{1}, y_{2}, y_{3}\right)=0$. Also define $u_{2}: X \rightarrow \mathbb{R}$ such that $u_{2}\left(x_{1}, x_{2}, x_{3}\right)=0, u_{2}\left(y_{1}, x_{2}, x_{3}\right)=u_{2}\left(x_{1}, y_{2}, x_{3}\right)=u_{2}\left(x_{1}, x_{2}, y_{3}\right)=3, u_{2}\left(x_{1}, y_{2}, y_{3}\right)=$ $u_{2}\left(y_{1}, y_{2}, x_{3}\right)=8, u_{2}\left(y_{1}, x_{2}, y_{3}\right)=12$, and $u_{2}\left(y_{1}, y_{2}, y_{3}\right)=20$. Now, $B\left(x_{1}, x_{2}, x_{3}\right)$ is empty since $B_{-1}\left(x_{1}, x_{2}, x_{3}\right)=\left\{\left(x_{1}, x_{2}, x_{3}\right),\left(y_{1}, x_{2}, x_{3}\right)\right\}$ and $B_{-2}\left(x_{1}, x_{2}, x_{3}\right)=\left\{\left(y_{1}, x_{2}, y_{3}\right),\left(y_{1}, y_{2}, y_{3}\right)\right\}$.

Thus, one needs further assumptions to obtain the nonempty-valuedness of $B$. 
Proof. For every $i \in N$, the coalitional best response correspondence of $-i$ is nonempty-valued. Utilizing symmetry in preference orders, we show that this property is carried over to the joint best response correspondence. To prove this, take any $i \in N$ and any $x \in B_{-i}(y)$. Also, take an arbitrary $j \in N \backslash\{i\}$. Given that $y_{i}=a$, this implies $u_{i}\left(a, x_{-i}\right) \geq u_{i}\left(a, x_{-i}^{\prime}\right)$ for every $x_{-i}^{\prime} \in X_{-i}$. In other words, given that $x_{j}=b$,

$$
\left\{z \in X \mid z_{i}=a\right\} \subset L\left(\left(x_{1}, \ldots, x_{i-1}, a, x_{i+1}, \ldots, x_{j-1}, b, x_{j+1}, \ldots, x_{|N|}\right), u_{i}\right) .
$$

By symmetry in preference orders, we have

$$
\left\{z \in X \mid z_{j}=a\right\} \subset L\left(\left(x_{1}, \ldots, x_{i-1}, b, x_{i+1}, \ldots, x_{j-1}, a, x_{j+1}, \ldots, x_{|N|}\right), u_{j}\right)
$$

implying that $u_{j}\left(a, x_{-j}\right) \geq u_{j}\left(a, x_{-j}^{\prime}\right)$ for every $x_{-j}^{\prime} \in X_{-j}$. Therefore, $x \in B_{-j}(y)$ as well. In fact, we have $x \in B(y)$ since $j$ is arbitrarily chosen; i.e., $B$ is nonemptyvalued.

In a similar way as in the proof of Theorem 3, it follows that $B$ is Veinottincreasing, and that $B(x)$ is a subcomplete sublattice for every $x \in X$. A Bergemodular game is a GSC à la Berge. Theorem 1 applies. The results follow.

At this point, there are two remarks to be emphasized. First, in above definitions, it is assumed for every $i \in N$ that $u_{i}$ is supermodular in $x_{-i}$, but not in $x_{i}$. Hence, opponent-wise supermodular games and Berge-modular games are not necessarily supermodular. Second, the proof of Theorem 4 utilizes symmetry in preference orders only for optimal strategies. Hence, it is possible to weaken the condition and to extend the existence result for a wider class of games.

Beside the existence, the result that the set of equilibrium is a complete lattice is essential in order to understand the order structure of the set of equilibrium. Moreover, since we utilize Zhou's [1994] fixed point theorem, we can refer to Echenique's [2005] constructive proof. This constructive approach indicates a way to compute the extremal Berge equilibria, that is the greatest and the lowest equilibria. Additionally, as the following theorem states, another result of the complementarity literature is valid for the set of Berge equilibrium. This result is a comparative statics property, a well appreciated property for the equilibrium set.

Theorem 5. Let $T$ be a partially ordered set, and $\left(G^{t}\right)_{t \in T}$ be a collection of $G S C$ à la Berge. Define $\mathcal{B}: X \times T \rightarrow X \times T$ to be the joint best response correspondence in $G^{t}$ such that for every $(x, t), \mathcal{B}(x, t)$ is the set of all $(y, t)$ where $y \in B(x)$. If $\mathcal{B}$ is Veinott increasing in $(x, t)$ on $X \times T$, and $\mathcal{B}(x, t)$ is a subcomplete sublattice of $X \times T$ for every $(x, t)$, then the extremal equilibria are increasing in $t$ on $T$.

Proof. We already know that the joint best response correspondence of each game $G^{t}$ satisfies the assumptions of Topkis's [1998] theorem restated above. Thus, the extremal equilibria are increasing in $t$ on $T$. 
We recall the aforementioned examples from the beginning of this section, and assume for every $i \in N$ that $x_{i}<y_{i}<z_{i}$. Then both examples are opponentwise supermodular. As for Berge-modular games, below are two examples in which $A<B$. It is worth noting that, in all four examples, Berge equilibrium makes more precise predictions.

$P l .3$

\begin{tabular}{|c|c|c|c|c|c|c|c|}
\hline \multirow[b]{3}{*}{ Pl.1 } & & \multicolumn{2}{|c|}{$A$} & & \multicolumn{2}{|c|}{$B$} \\
\hline & $B$ & $1,1,1$ & $1,1,1$ & & $B$ & $1,1,1$ & $2,2,2$ \\
\hline & $A$ & $1,1,1$ & $1,1,1$ & Pl.1 & $A$ & $1,1,1$ & $1,1,1$ \\
\hline & & $\begin{array}{c}A \\
P l .2\end{array}$ & $B$ & & & $\begin{array}{c}A \\
P l .2\end{array}$ & $B$ \\
\hline
\end{tabular}

In the above example, Berge equilibrium predicts $\{(A, A, A),(B, B, B)\}$ which is included in the set of Nash equilibrium. In the following example, $\{(A, A, A),(B, B, B)\}$ is the set of Nash equilibrium. However, the only Pareto dominating strategy profile is the unique Berge equilibrium: $(B, B, B)$,

\begin{tabular}{|c|c|c|c|c|c|c|c|}
\hline & & & & & & & \\
\hline & $B$ & $0,2,3$ & $1,1,8$ & & $B$ & $1,4,2$ & $4,4,8$ \\
\hline Pl.1 & $A$ & $0,0,0$ & $2,0,3$ & Pl.1 & $A$ & $2,2,0$ & $4,1,2$ \\
\hline & & $\begin{array}{c}A \\
P l .2\end{array}$ & $B$ & & & $\begin{array}{c}A \\
P l .2\end{array}$ & $B$ \\
\hline
\end{tabular}

\section{Conclusion}

Providing a sufficient condition under which the joint best response correspondence is nonempty-valued, this paper studies the existence of Berge equilibrium for Bergemodular games and for GSC à la Berge. We prove that the equilibrium set is a nonempty complete lattice and that it satisfies the Topkis' monotone comparative statics property.

\section{Acknowledgments}

We thank Emin Karagözoğlu, an associate editor, and two reviewers for helpful comments and suggestions.

\section{References}

Abalo, K. Y. and Kostreva, M. M. [2004] Some existence theorems of Nash and Berge equilibria, Appl. Math. Lett. 17, 569-573.

Abalo, K. Y. and Kostreva, M. M. [2005] Berge equilibrium: Some recent results from fixed-point theorems, Appl. Math. Comput. 169, 624-634. 
Arnsperger, C. and Varoufakis, Y. [2003] Toward a theory of solidarity, Erkenntnis 59(2), $157-188$.

Berge, C. [1957] Théorie Générale des Jeux à n Personnes (Gautier Villars, Paris).

Bolton, G. E. and Ockenfels, A. [2000] ERC: A theory of equity, reciprocity, and competition, Amer. Econ. Rev. 90, 166-193.

Charness, G. and Rabin, M. [2002] Understanding social preferences and simple tests, Quart. J. Econ. 117(3), 817-869.

Colman, A. M., Körner, T. W., Musy, O. and Tazdaït, T. [2011] Mutual support in games: Some properties of Berge equilibria, J. Math. Psychol. 55, 166-175.

Echenique, F. [2005] A short and constructive proof of Tarski's fixed-point theorem, Int. J. Game Theory 33(2), 215-218.

Fehr, E. and Schmidt, K. M. [1999] A theory of fairness, competition, and cooperation, Quart J. Econ. 114(3), 817-868.

Forsythe, R., Horowitz, J., Savin, N. E. and Sefton, M. [1994] Fairness in simple bargaining experiments, Games Econ. Behav. 6, 347-369.

Güth, W., Schmittberger, R. and Schwarze, B. [1982] An experimental analysis of ultimatum bargaining, J. Econ. Behav. Organ. 3(4), 367-388.

Larbani, M. and Nessah, R. [2008] A note on the existence of Berge and Berge-Nash equilibria, Math. Soc. Sci. 55, 258-271.

Musy, O., Pottier, A. and Tazdaï, T. [2012] A new theorem to find Berge equilibria, Int. Game Theor. Rev. 14(1), 1250005.

Nessah, R., Larbani, M. and Tazdaït, T. [2007] A note on Berge equilibrium, Appl. Math. Lett. 20, 926-932.

Tarski, A. [1955] A lattice-theoretical fixpoint theorem and its applications, Pacific J. Math. 5, 285-309.

Topkis, D. M. [1998] Supermodularity and Complementarity (Princeton University Press, Princeton).

Vives, X. [2005] Complementarities and games: New developments, J. Econ. Lit. 43, $437-479$.

Zhou, L. [1994] The set of Nash equilibria of a supermodular game is a complete lattice, Games Econ. Behav. 7, 295-300.

Zhukovskii, V. I. [1994] Linear Quadratic Differential Games (Naoukova Doumka, Kiev). 